\title{
Model-based fault diagnosis and tolerant control: the ESA's e.Deorbit mission
}

\author{
D. Henry ${ }^{1}$, J. Cieslak ${ }^{1}$, J. Zenteno Torres ${ }^{1}$, P. Colmenarejo ${ }^{2}$, J. Branco ${ }^{3}$, N. Santos ${ }^{3}$, P. Serra ${ }^{3,9}$, \\ J. Telaard ${ }^{4}$, H. Strauch ${ }^{4}$, A.M. Giordano ${ }^{5}$, M. De Stefano ${ }^{5}$, C. Ott ${ }^{5}$, M. Reiner ${ }^{5}$, J. Jaworsk ${ }^{6}$, E. Papadopoulos ${ }^{7}$, \\ G. Visentin ${ }^{8}$, F. Ankersen ${ }^{8}$, J.G. Fernandez ${ }^{8}$
}

\begin{abstract}
The ESA (European Space Agency) is currently pursuing the development of the e.Deorbit mission that will remove a large defunct satellite from Earth orbit: ENVISAT. To fulfil the mission autonomy requirements, ESA has decided to embed in the GNC (Guidance, Navigation, Control) software, fault tolerance capacities against actuator faults. The aim of this paper is to present the development and validation of a model-based fault diagnosis and tolerant control solution for such faults. The proposed solution is based on a new class of nonlinear unknown input observers, optimal in the $L_{2}$-gain sense, and a modified version of the nonlinear inverse pseudo control allocation technique. An intensive simulation campaign conducted within a high-fidelity nonlinear industrial simulator, demonstrates the efficiency of the approach.
\end{abstract}

\section{INTRODUCTION}

The ESA (European Space Agency) is currently pursuing the development of the e.Deorbit mission that will remove a large $(8.2$ tons, $26 \mathrm{~m} \times 10 \mathrm{~m} \times 5 \mathrm{~m})$ defunct satellite from the Earth orbit: ENVISAT. The e.Deorbit mission pertains to a particular technological means for active debris removal that involves a seven degree of freedom robotic manipulator, being operated in tight coordination with the chaser platform motion, see Fig. 1 for an illustration. This particular technological context motivated ESA to manage studies to develop fault detection, isolation (FDI) and tolerant control (FTC) solutions for the propulsion unit of the chaser platform.

${ }^{1}$ IMS Lab., Univ. Bordeaux, Bordeaux INP, CNRS, 351 Cours de la Libération, 33405 Talence, France (david.henry,jerome.cieslak@u-bordeaux.fr)

${ }^{2}$ GMV, Isaac Newton 11, P.T.M. Tres Cantos, E-28760 Madrid, Spain, (pcolmena@gmv.com)

${ }^{3}$ GMV Skysoft, Av. D. João II, n. 43, Torre Fernão de Magalhães $7^{\circ}, 1998-025$ Lisboa, Portugal, (jbranco,nuno.santos,parroz@gmv.com)

${ }^{4}$ Airbus Defence and Space, Airbus Allee 1, 28199 Bremen, Germany, (juergen.telaar,hans.strauch@ airbus.com)

${ }^{5}$ Deutsches Zentrum für Luft- und Raumfahrt (DLR e.V.), Institute of Robotics and Mechatronics, 82234, Wessling, Germany (Alessandro.Giordano,Marco.DeStefano,Christian.Ott,Matthias.Reiner@dlr.de)

${ }^{6}$ Przemyslowy Instytut Automatyki i Pomiarów (PIAP), Jerozolimskie 202, 02-486 Warsaw, Poland, (jaroslaw.jaworski@ piapspace.com)

${ }^{7}$ NTUA, 9 Heroon Polytechneiou, GR- 15780 Zografou, Athens, Greece, (egpapado@central.ntua.gr)

${ }^{8}$ ESA/ESTEC, Keplerlaan 1, 2201 AZ Noordwijk, Netherlands, (Gianfranco.Visentin,Finn.Ankersen,jesus.gil.fernandez@esa.int)

${ }^{9}$ Lusofona University of Humanities and Technologies ULHT, ECATI, COPELABS, Lisbon, Portugal
Numerous model-based FDI techniques have been developed in the academic community. With regards to the problem of spacecraft thruster faults, one can mention the work presented in [1] that proposes a sliding mode observerbased approach for the Mars Express spacecraft during the sun acquisition mode. The work reported in [13] addresses too the Mars Express experiment and is based on both state estimation and unknown input decoupling. In [15], an extended Kalman filter is used to estimate the abnormal (fault) torque. The Mars Sample Return mission is considered in [8]. A solution is developed based on the $H_{\infty} / H_{-}$theory and a bank of unknown input observers jointly used with a crosscorrelation test for fault isolation. The $H_{\infty} / H_{-}$approach is considered for micro-Newton colloidal thruster faults during the experiment phase for the LISA Pathfinder experiment in [3] and in [6], $H_{\infty} / H_{-}$filter-based strategies are also proposed to diagnose thruster faults for the Microscope satellite. Thruster faults during station keeping manoeuvres are considered for telecom satellites in [2]. A pure $H_{\infty}$ FDI strategy is proposed in [11] for control surface faults in the Hopper re-entry vehicle and [14] proposed some solutions for MYRIADE microsatellites thruster faults.

With regards to FTC solutions, the control allocation technique (see [10] for a survey) has been implemented using a SIMPLEX-based algorithm in the Automated Transfer Vehicle that has docked five times with the International Space Station. [12] proposed a retreat strategy at the guidance level to accommodate faults occurring in the actuation system of the chaser of the Mars Sample Return mission, during in orbit rendezvous with a canister.

Note that this overview of existing works covers only studies that deal with real space missions. There exists plenty of papers that addresses the design of FDI-FTC solutions for spacecraft but they are thought to be more or less, an academic exercise since they are not representative of the full problem (in view of the authors of this paper). Often, the spacecraft is considered as a rigid body in which the coupling between the rotational and translational motions, the effect of flexible appendages or the effect of propellant sloshing are not considered. This paper overcomes this problem. The proposed strategy follows the study presented in [4], i.e. NL-UIO and control allocation techniques are used to diagnose and accommodate thruster faults. The major difference results in the NL-UIO technique. 


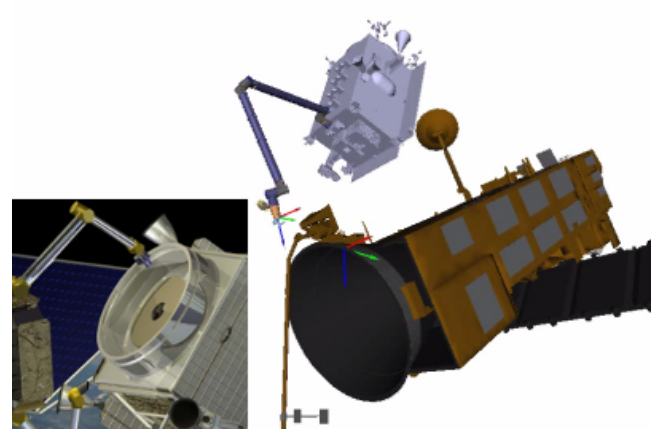

Fig. 1. e.Deorbit mission concept

\section{REFERENCE SCENARIO}

The e.Deorbit mission involves several phases, namely synchronisation, capture, rigidisation and stabilisation phases. The synchronisation starts with a transition of the chaser from Parking Hold Point to Capture Point. The motion synchronisation strategy comprises several steps, i.e. V-bar approach from 100 to $30 \mathrm{~m}$, spherical fly-around to target angular momentum vector, approach along angular momentum vector, synchronisation of rotational motion and transfer to the capture point. At the capture point, a robot arm moves the open gripper to the grasping point, located on the launcher adapter ring, see Fig. 1 for an illustration. As soon as the gripper is in the right position for grasping, it closes quickly, establishing a form closure assuring that ENVISAT cannot escape (soft capture). After confirmation of successful capture, the robot arm is rigidised (hard capture). The stabilisation of the coupled system (chaser plus target) begins upon confirmation of arm rigidisation.

The GNC (Guidance Navigation and Control) and avionics architecture retained for the mission, is composed of a LIDAR which allows full relative pose estimation, an IMU (accelerometer and gyro), 3 Star Tracker heads, a Sun Sensor, a GPS receiver and a GPS constellation propagation. The actuation consists of 24 thrusters of $22 N$ for both attitude and position control. The robot arm is equipped with 7 joints, a gripper and a vision system for relative navigation between gripper and grasping point. The combined controller for the chaser satellite and the robotic arm, has been designed using $H_{\infty}$ techniques in order to guarantee robust performance and stability. The controller issues simultaneously $i$ ) force and torque commands for the chaser, and, $i i$ ) manipulator joint torque commands which are realised by the inner joint control loops.

The complete mission is modelled into a so-called functional engineering simulator developed in Matlab/Simulink, within the library called SPACELAB that contains e.g. sensors, actuators, dynamic, kinematic and environment models. Both chaser and target have each one an associated environment module as some characteristics depend on specific spacecraft properties. Typically, the chaser has two partially filled tanks that cause propellant sloshing, whereas it is assumed that the tanks of ENVISAT are empty. A contrario, since ENVISAT has still its solar arrays deployed, flexible modes are considered. For both spacecraft, the considered disturbances are central body acceleration, non-spherical gravity acceleration, gravity gradient torque, solar radiation pressure, third-body perturbation (Moon and Sun), Earth magnetic torque and aerodynamic drag. The implementation of the multi-body dynamics model of the robot arm has been carried out based on the Simscape Multibody technology. A universe library provides the ephemerides of Earth, Moon, and Sun.

\section{THE FDI UNIT}

\section{A. Modelling issues}

Recent studies [4], [8] demonstrated that thrusters faults can be successfully diagnosed using the model that links the actuator (thruster) commands with the IMU measurement. This approach is retained in this work. Then, by virtue of the Euler's second law, it follows (in the so-called body frame):

$$
\dot{\omega}=J^{-1} \sum_{k} T_{k}-J^{-1} \omega \times J \omega
$$

$\omega \in \mathbb{R}^{3}$ is the angular velocity vector and $J \in \mathbb{R}^{3 \times 3}$ is the inertia dyadic about the center of mass CoM. $\sum_{k} T_{k}=T_{s}+$ $T_{p}+T_{d}$ describes the sum of torques about CoM. $T_{s} \in \mathbb{R}^{3}$ refers to the torques induced by propellant sloshing, $T_{p} \in$ $\mathbb{R}^{3}$ refers to the torques caused by the propulsion unit and $T_{d} \in \mathbb{R}^{3}$ is considered to be sum of the disturbance described in the previous section and the reaction torques due to the robotic arm motion. Note that in this work, $T_{d}$ is considered as a general disturbance vector.

Propellant sloshing in each tank is modelled as a linear 3D spring-mass model that considers the Coriolis, the centrifugal and the Euler accelerations and of course, the accelerations due to the actuation unit and disturbances, see [8] for details if necessary. Merging the sloshing model of each tank into a unique state space model leads to the equations

$$
\begin{aligned}
& \dot{x}_{s}=A_{s} x_{s}+\left[\begin{array}{lll}
\frac{1}{m} B_{s F} & B_{s \omega} & B_{s \dot{\omega}}
\end{array}\right]\left[\begin{array}{c}
F_{s}+F_{p}+F_{d} \\
\omega \\
\dot{\omega}
\end{array}\right] \\
& {\left[\begin{array}{l}
F_{s} \\
T_{s}
\end{array}\right]=\left[\begin{array}{l}
C_{s F} \\
C_{s T}
\end{array}\right] x_{s}}
\end{aligned}
$$

where $F_{s} \in \mathbb{R}^{3}, F_{p} \in \mathbb{R}^{3}$ and $F_{d} \in \mathbb{R}^{3}$ refer to the forces induced by propellant sloshing in the two tanks, the forces caused by the thruster-based propulsion unit and the disturbance forces (considered unknown but with an a priori known distribution matrix), all given in the body frame. $m$ and $x_{s} \in \mathbb{R}^{36}$ refer to the chaser's mass and the state vector associated to the sloshing modes of the two tanks. $A_{s}, B_{s F}, B_{s \omega}, B_{s \dot{\omega}}, C_{s F}, C_{s T}$ are matrices that depend on the sloshing characteristics given in table I.

Combining the models (1) and (2)-(3) into a unique state space representation leads to

$$
\begin{aligned}
& \dot{x}=A x+\phi(\omega)+B\left[\begin{array}{l}
F_{p} \\
T_{p}
\end{array}\right]+E\left[\begin{array}{l}
F_{d} \\
T_{d}
\end{array}\right] \\
& y=\left[\begin{array}{ll}
\mathbb{O} & \mathbb{I}_{3}
\end{array}\right] x
\end{aligned}
$$




\begin{tabular}{r|c|c|c|c|c} 
slosh models & damping coeff. & frequency of mode 1 & frequency of mode 2 & frequency of mode 3 & propellant mass \\
\hline Tank 1 & $0.1 \pm 50 \%$ & $0.478 \mathrm{rd} / \mathrm{s} \pm 2 \%$ & $0.821 \mathrm{rd} / \mathrm{s} \pm 2 \%$ & $1.04 \mathrm{rd} / \mathrm{s} \pm 2 \%$ & $13.8 \mathrm{~kg} \pm 2 \%$ \\
Tank 2 & $0.1 \pm 50 \%$ & $0.478 \mathrm{rd} / \mathrm{s} \pm 2 \%$ & $0.821 \mathrm{rd} / \mathrm{s} \pm 2 \%$ & $1.04 \mathrm{rd} / \mathrm{s} \pm 2 \%$ & $22.1 \mathrm{~kg} \pm 2 \%$ \\
\hline \hline
\end{tabular}

TABLE I

LIST OF UNCERTAINTIES.

where $\mathbb{I}, \mathbb{O}$ stand for the identity matrix and the null matrix of adequate dimension, respectively. The augmented state vector $x$ is defined according to $x=\left[x_{s}^{T} \omega^{T}\right]^{T}$. The matrices $A, B$ and the nonlinear function $\phi(\omega)$ are:

$$
\begin{gathered}
A=\left[\begin{array}{cc}
A_{s}+\frac{1}{m} B_{s F} C_{s F}+B_{s \dot{\omega}} J^{-1} C_{s T} & \mathbb{O} \\
J^{-1} C_{s T} & \mathbb{O}
\end{array}\right] \\
\phi(\omega)=\left[\begin{array}{c}
B_{s \omega} \omega-B_{s \dot{\omega}} J^{-1}(\omega \times J \omega) \\
-J^{-1}(\omega \times J \omega)
\end{array}\right] \\
B=\left[\begin{array}{cc}
\frac{1}{m} B_{s F} & B_{s \dot{\omega}} J^{-1} \\
\mathbb{O} & J^{-1}
\end{array}\right], E=B
\end{gathered}
$$

Let $\mathcal{S}_{\text {all }}=\{1, \ldots, 24\}$ denote the set of all the thruster indices, and let $u_{k}(t), \forall k \in \mathcal{S}_{\text {all }}$ be the commanded opening duration of the $k t h$ thruster. The torques $T_{p}$ and forces $F_{p}$ generated by thrusters are given by (fault free cases)

$$
\left[\begin{array}{l}
F_{p} \\
T_{p}
\end{array}\right]=\left[\begin{array}{c}
M_{F} \\
M_{T}
\end{array}\right] u(t)=M u(t), \quad M \in \mathbb{R}^{6 \times 24}
$$

The columns of $M$ (called the thruster configuration matrix) are the influence coefficients defining how each thruster affects each component of $T_{p}$ and $F_{p}$. With regard to the thruster faults considered in this work, the focus is on the stuck open (fully open) and stuck closed (closed thruster) faults. The following mathematical model can be used to describe these faults:

$$
\phi_{k}(t)= \begin{cases}\max \left\{u_{k}(t), 1\right\} & \text { if stuck open } \\ 0 & \text { if stuck closed }\end{cases}
$$

where the index $k$ refers to the $k t h$ thruster. Assuming no simultaneous faults, the considered thruster faults can be modeled in a multiplicative way according to (the index $f$ outlines the faulty case)

$$
u_{f}(t)=\left(\mathbb{I}_{24}-\Psi(t)\right) u(t)
$$

with $\Psi(t)=\operatorname{diag}\left(\psi_{1}(t), \ldots, \psi_{24}(t)\right)$, where $0 \leq \psi_{k}(t) \leq$ $1, \forall k \in \mathcal{S}_{\text {all }}$ are unknown. The status of the $k t h$ thruster is modeled by $\psi_{k}$ as follows:

$$
\psi_{k}(t)= \begin{cases}0 & \text { if healthy } \\ 1-\phi_{k}(t) / u_{k}(t) & \text { if faulty }\end{cases}
$$

where $\phi_{k}$ enables to consider the different fault scenarios.

Combining the equations (4), (8) and (10) together, leads to the following state space representation that models both the fault-free and faulty rotational dynamics of the chaser, the measurement $y$ being provided by the IMU:

$$
\begin{aligned}
& \dot{x}=A x+\phi(\omega)+B M\left(\mathbb{I}_{24}-\Psi(t)\right) u(t)+E\left[\begin{array}{l}
F_{d} \\
T_{d}
\end{array}\right] \\
& y=\left[\begin{array}{ll}
\mathbb{O} & \mathbb{I}_{3}
\end{array}\right] x
\end{aligned}
$$

Then, using an additive approximation [5] of the fault multiplicative fault model stated by (9)-(11), (12) can be re-written according to

$$
\begin{aligned}
& \dot{x}=A(\rho) x+\phi(\omega, \rho)+B(\rho) M(\rho) u+E(\rho)\left[\begin{array}{c}
F_{d} \\
T_{d}
\end{array}\right]+K(\rho) f \\
& y=\left[\begin{array}{ll}
\mathbb{O} & \mathbb{I}_{3}
\end{array}\right] x
\end{aligned}
$$

In this equation, the $k t h$ column of the matrix $K$ is the $k t h$ fault signature associated to the $k t h$ fault mode $f_{k}$. The indices $k=1, \ldots, 24$ also coincide with the numbering of components of the set $S_{\text {all }}$, and thus with the columns of the matrix $M$, see (8).

This model depends nonlinearly on the parameters listed in table I, that are gathered in (13) into the vector $\rho$. It seems obvious that many of these parameters vary during the mission such as $m$ and CoM due to e.g. propellant consumption. Thus, $\rho$ is a time varying vector.

Using the higher-order singular value decomposition canonical form of tensor product model transformation, a finite set of bounded hyper-parameters $\pi_{i}(t), i=1, \ldots N_{p}$ : $\pi(t)=\left[\pi_{1}(t), \ldots, \pi_{N_{p}}(t)\right],\left|\pi_{i}(t)\right| \leq \bar{\pi}_{i}$ is deduced from the domain of variation of $\rho(t)$, so that

$\dot{x}=A(\pi) x+\phi(\omega, \pi)+B(\pi) M(\pi) u+E(\pi)\left[\begin{array}{c}F_{d} \\ T_{d}\end{array}\right]+K(\pi) f$ $y=\left[\begin{array}{ll}\mathbb{O} & \mathbb{I}_{3}\end{array}\right] x$

and so that components $\pi_{i}$ enter the model (14) in a affine manner, see [7] for details about this technique. $\pi(t)$ is assumed to be available in real time and to lie in the polytope $\Xi$ of vertices $\Pi_{i}, i=1, \ldots, N_{v}$, satisfying the convex decomposition

$$
\pi=\beta_{1} \Pi_{1}+\ldots+\beta_{N_{v}} \Pi_{N_{v}}, \quad \beta_{i} \geq 0, \quad \sum_{i=1}^{N_{v}} \beta_{i}=1
$$

Note that as explained in [7], $\Xi$ is a polytope that overapproximates the domain of $\rho$. Then, (14) is more conservative than (13).

Remark 1: It is clear that the definition of $\pi$ plays a central role in the proposed approach. Due to space limitation, the definition of $\pi$ is not given in this paper. Each $\pi_{i}, i=1, \ldots N_{p}$ is in fact, a functional of some chaser properties that are estimated in real-time by the navigation unit.

\section{B. The FDI unit}

The FDI strategy consists of two main functions, namely decision making which provides alarm generation and fault isolation which circumscribes the fault to elements of $\mathcal{S}_{\text {all }}$. The proposed solution here involves a bank of nonlinear unknown input observers (NL-UIOs), built upon Eq. (14). 
1) Fault isolability discussion:: Let us first come back to the fault distribution matrix $K(\pi)$ in (14). Analysing $K(\pi)$ in terms of directions spanned by its column vectors, reveals that $\mathcal{S}_{\text {all }}$ can be further classified into the following subsets

$\mathcal{S}_{1}=\{1,2\}, \mathcal{S}_{2}=\{3,4\}, \mathcal{S}_{3}=\{5,7\}, \mathcal{S}_{4}=\{6,8\}$

$\mathcal{S}_{5}=\{13,14\}, \mathcal{S}_{6}=\{15,16\}, \mathcal{S}_{7}=\{17,19\}, \mathcal{S}_{8}=\{18,20\}$

so that, for indices in a given set $\mathcal{S}_{k}$, the associated column vectors of $K(\pi)$ are colinear. This clearly demonstrates that it is not possible to distinguish a fault occurring in a given pair of thrusters associated to a set $\mathcal{S}_{k}$ by means of decoupling approaches like NL-UIOs. This property can be mathematically formalised as the following rank deficiency property: for a given set $\mathcal{S}_{k}, k=1, \ldots, 8$,

$$
\operatorname{rank}\left[T_{k}(\pi) K(\pi)\right] \neq \operatorname{rank}[K(\pi)] \quad \forall \pi \in \Xi
$$

where $T_{k}$ refers to the decoupling matrix of the $k t h$ NL-UIO, as it will be defined later, see (26). This is a limitation of the proposed solution. A solution to this problem may result in using the techniques discussed in, e.g. [7].

Additionally, we define the following sets:

$$
\begin{aligned}
& \mathcal{S}_{9}=\{9\}, \mathcal{S}_{10}=\{10\}, \mathcal{S}_{11}=\{11\}, \mathcal{S}_{12}=\{12\} \\
& \mathcal{S}_{13}=\{21\}, \mathcal{S}_{14}=\{22\}, \mathcal{S}_{15}=\{23\}, \mathcal{S}_{16}=\{24\}
\end{aligned}
$$

2) NL-UIO design:: Thanks to the definition of the sets $\mathcal{S}_{k}, k=1, \ldots, 16$, we are now able to well pose the design of the FDI unit.

Problem 1: Consider the model (14). The problem consists of the design of a bank of 16 NL-UIOs so that the output estimation error $e_{y_{k}}(t)=\hat{y}_{k}(t)-y(t) \in \mathbb{R}^{3}$ of the $k t h$ NL-UIO is decoupled from the faults involved in the set $\mathcal{S}_{k}$ while achieving robustness against disturbances $F_{d}, T_{d}$ $\forall \pi(t) \in \Xi$, in the $L_{2}$-gain sense. $y(t)$ is the measured angular velocity $\omega(t)$ provided by the IMU sensor and $\hat{y}_{k}(t)$ is the estimate provided by the $k t h$ NL-UIO. Then, the UIO with the minimum estimation error in the sense of the $L_{2}$-norm, i.e. $\min _{k}\left\|e_{y_{k}}\right\|_{2}, k=1, \ldots, 16$, reveals that a fault occurs in the thrusters associated to $\mathcal{S}_{k}$.

As an illustration case, we consider now the case of designing the first NL-UIO, i.e. we focus on $\mathcal{S}_{1}$. To proceed, $f$ is split into two subsets according to the definition of $\mathcal{S}_{1}$ and $\mathcal{S}_{j}, j=2, \ldots, 16$, namely $\bar{f}=\left[f_{1} f_{2}\right]^{T}$ which will play the role of the unknown input vector to be decoupled and $\underline{f}=\left[f_{3} \ldots f_{24}\right]^{T}$ which are the remaining components of $\bar{f}$. The partition of $K(\pi)$ follows so that (14) is rewritten according to (the dependency of $\pi$ is omitted for clarity)

$$
\begin{aligned}
\dot{x} & =A x+\phi(\omega)+B M u+E d+\overline{K f}+\underline{K} \underline{f} \\
y & =\left[\begin{array}{ll}
\mathbb{O} & \mathbb{I}_{3}
\end{array}\right] x=C x
\end{aligned}
$$

where $d=\alpha\left[F_{d}^{T} T_{d}^{T}\right]^{T} . \alpha$ is a diagonal matrix of positive elements, i.e. $\alpha=\operatorname{diag}\left(\alpha_{i}\right): \alpha_{i} \geq 0, i=1, \ldots, 6$, that is introduced to weight the $L_{2}$-gain problem. By such a parametrisation, it is possible to penalise some components of $d$ within the $L_{2}$-gain optimisation problem. This has been revealed useful in practice in order to not decrease the sensitivity level of the NL-UIO against $f$.

Then, the goal is to design the following NL-UIO

$$
\begin{aligned}
& \dot{z}=N(\pi) z+T(\pi) \phi_{\varepsilon}+H(\pi) u+L_{1}(\pi) y \\
& \hat{x}=z-L_{2}(\pi) y \\
& e_{y}=\hat{y}-y=C \hat{x}-y
\end{aligned}
$$

with $\phi_{\varepsilon}=\phi(\varepsilon \operatorname{sat}(\omega), \pi)$, in such a way that, $\forall \pi \in \Xi, e_{y}$ is decoupled from $\bar{f}$ and robust to $d$, in the $L_{2}$-gain sense. The solution to this problem is given in the following.

Theorem 1: Assume that

$$
\left\|\phi_{\varepsilon}-\phi(\omega, \pi)\right\| \leq \mu\|\hat{x}-x\|
$$

The NL-UIO given by (20)-(22) exists if there exist a Lyapunov matrix $X=X^{T}>0$, matrices $\bar{Y}_{j}, \bar{Z}_{j}, j=1, \ldots, N_{v}$ of adequate dimension and a scalar $\gamma$ so that (24) is satisfied. In (24), (. $)^{\dagger}$ stands for the Moore-Penrose inverse of matrix and the notation $\mathcal{M}_{j}$ refers to the matrix $\mathcal{M}(\pi)$ evaluated at the vertex $\Pi_{j}$, i.e. $\mathcal{M}_{j}=\mathcal{M}\left(\Pi_{j}\right)$.

Then (the dependency of $\pi$ is again omitted for clarity):

$$
\begin{aligned}
& N=\left(\mathbb{I}+L_{2} C\right) A-Z C \\
& T=\left(\mathbb{I}+L_{2} C\right), \quad H=T B M \\
& L_{1}=Z\left(\mathbb{I}+C L_{2}\right)-\left(\mathbb{I}+L_{2} C\right) A L_{2} \\
& L_{2}=U+Y V, \quad Y=X^{-1} \bar{Y}, \quad Z=X^{-1} \bar{Z}
\end{aligned}
$$

and the (quadratic) $L_{2}$-gain performance level between $e_{y}$ and $d$ is less or equal to $\gamma$. In (25)-(28), all " $\pi$-depend" matrices are deduced from their vertex values using the convex decomposition (15).

Proof : First, note that due to its definition, $\bar{K}(\pi)$ is of full column rank and $\operatorname{rank}[C \bar{K}(\pi)]=\operatorname{rank}[\bar{K}(\pi)], \forall \pi \in$ $\Xi$, which guarantees a solution to the problem. Next, and as usual in UIO theory, the design of the NL-UIO is done without considering $f$, so $f=0$.

Consider now the estimation error $e=\hat{x}-x$. Then, with (25)-(28) (the dependency of $\pi$ is omitted for clarity)

$$
\dot{e}=N e+T\left(\phi_{\varepsilon}-\phi\right)-T E d-T \bar{K} f
$$

where $\phi=\phi(\omega, \pi)$. By virtue of the $L_{2}$-gain quadratic performance theory, the NL-UIO is quadratically stable and achieve $L_{2}$-gain quadratic performance level $\gamma$ if there exists a Lyapunov function $V=e^{T} X e: X=X^{T}>0$ so that $\dot{V}+e^{T} e-\gamma^{2} d^{T} d<0$.

It can be verified that

$$
\begin{aligned}
\dot{V} & =e^{T}\left(N^{T} X+X N\right) e+2 e^{T} X T\left(\phi_{\varepsilon}-\phi\right) \\
& -d^{T} E^{T} T^{T} X e-e^{T} X T E d \\
\dot{V} & \leq e^{T}\left(N^{T} X+X N\right) e+2\left\|e^{T} X T\right\|\left\|\phi_{\varepsilon}-\phi\right\| \\
& -d^{T} E^{T} T^{T} X e-e^{T} X T E d
\end{aligned}
$$

and then, with (23)

$$
\begin{aligned}
\dot{V} & \leq e^{T}\left(N^{T} X+X N+\mu\left(\mathbb{I}+X T T^{T} X\right)\right) e \\
& -d^{T} E^{T} T^{T} X e-e^{T} X T E d
\end{aligned}
$$




$$
\left[\begin{array}{ccc}
P_{j}+P_{j}^{T}+(\mu+1) \mathbb{I} & -\left(X\left(\mathbb{I}+U_{j} C\right)+\bar{Y}_{j} V_{j} C\right) E_{j} & \sqrt{\mu}\left(X\left(\mathbb{I}+U_{j} C\right)+\bar{Y}_{j} V_{j} C\right) \\
-\left(\left(X\left(\mathbb{I}+U_{j} C\right)+\bar{Y}_{j} V_{j} C\right) E_{j}\right)^{T} & -\gamma^{2} \mathbb{I} & \mathbb{O} \\
\sqrt{\mu}\left(X\left(\mathbb{I}+U_{j} C\right)+\bar{Y}_{j} V_{j} C\right)^{T} & \mathbb{O} & -\mathbb{I}
\end{array}\right]<0, \quad j=1, \ldots, N_{v}
$$

$$
P_{j}=X\left(\mathbb{I}+U_{j} C\right) A_{j}+\bar{Y}_{j} V_{j} C A_{j}-\bar{Z}_{j} C, \quad U_{j}=-\bar{K}_{j}\left(C \bar{K}_{j}\right)^{\dagger}, V_{j}=\mathbb{I}-C \bar{K}_{j}\left(C \bar{K}_{j}\right)^{\dagger}
$$

Then, $\dot{V}+e^{T} e-\gamma^{2} d^{T} d<0$ is equivalent to

$$
\left[\begin{array}{cc}
G+W W^{T} & -X T E \\
-E^{T} T^{T} X & -\gamma^{2} \mathbb{I}
\end{array}\right]<0
$$

with $G=N^{T} X+X N+(\mu+1) \mathbb{I}$ and $W=\sqrt{\mu} X T$. This inequality can be re-written according to

$$
\left[\begin{array}{cc}
G & -X T E \\
-E^{T} T^{T} X & -\gamma^{2} \mathbb{I}
\end{array}\right]+\left[\begin{array}{c}
W \\
\mathbb{O}
\end{array}\right]\left[\begin{array}{ll}
W^{T} & \mathbb{O}
\end{array}\right]<0
$$

and then, by congruence with $\left[\begin{array}{c|c}\mathbb{I} & {\left[\begin{array}{l}W \\ \mathbb{O}\end{array}\right]} \\ \hline \mathbb{O} & \mathbb{I}\end{array}\right]$, it follows

$$
\left[\begin{array}{cc|c}
G & -X T E & W \\
-E^{T} T^{T} X & -\gamma^{2} \mathbb{I} & \mathbb{O} \\
\hline W^{T} & \mathbb{O} & -\mathbb{I}
\end{array}\right]<0
$$

By virtue of the vertex property and with (25)-(28), (34) yields iff inequality (24) yields, which is a LMI in $X, \bar{Y}\left(\Pi_{j}\right), \bar{Z}\left(\Pi_{j}\right) j=1, \ldots, N_{v}$

3) Alarm generation: : For decision making, consider $r(t)$ defined according to $r(t)=\left\|\left[e_{y_{1}}(t) \ldots e_{y_{16}}(t)\right]^{T}\right\|_{2}$. The decision test results in the Boolean signal $\mathcal{B}(t)$ given by

$$
\mathcal{B}(t)=\left\{\begin{array}{l}
1 \text { if } \mathbb{E}\left\{r\left(t_{n}\right)\right\}>J_{m} \text { and/or } \mathbb{E}\left\{r^{2}\left(t_{n}\right)\right\}>J_{v} \\
0 \text { if } \mathbb{E}\left\{r\left(t_{n}\right)\right\} \leq J_{m} \text { and } \mathbb{E}\left\{r^{2}\left(t_{n}\right)\right\} \leq J_{v}
\end{array}\right.
$$

where $\mathbb{E}\{$.$\} refers to the expectation operator. r\left(t_{n}\right)$ refers to $r(t)$ evaluated at time instant $t=t_{n}=n T_{s}, n \in \mathbb{R}^{+}$, $T_{s}$ being the sampling period of the GNC. Additionally a confirmation time $t_{c}$ is considered, i.e. an alarm is generated iff $\mathcal{B}(t)=1$ during at least $t_{c}$.

\section{THE FTC UNIT}

The proposed FTC solution consists of control reallocation, thanks to the redundancy in the thruster configuration. The proposed strategy works as follows: as soon as the faulty thrusters are isolated by the FDI unit, they are turned off using dedicated thruster latch valves and the forces and torques computed by the controller are re-allocated among the healthy thrusters. This problem can be formulated as the following optimisation problem:

$$
u=\underset{u \in \mathcal{U}=\left\{u_{k}: 0 \leq u_{k} \leq \bar{u}_{k}\right\}, \forall k \in \mathcal{S}_{\text {all }}}{\operatorname{argmin}}\left\|W_{v}\left(M u-v_{r}\right)\right\|_{p}
$$

$v_{r}$ is the vector of the desired force and torque commands and $\bar{u}_{k}$ is the maximum opening value of the $k t h$ thruster. The basic of the fault tolerance principle is that if the kth thruster is declared faulty by the FDI unit, then $\bar{u}_{k}$ is set to "0". The nonsingular weighting matrix $W_{v}$ affects the prioritization among force/torque components.

The different choice of the vector $p$-norm results in $i$ ) minimum flow rate allocation for $p=1$, minimum power allocation for $p=2$ and $i i i)$ minimum peak torque/force allocation for $p=\infty$. Here, $p=1$ has been revealed to lead to the smallest propellant consumption.

Algorithm 1, whose foundations come from the nonlinear pseudo-inverse controller technique initially formulated by [9], states the algorithm that solves (36). The algorithm terminates if the precision $\epsilon$ of the allocated torques/forces, weighted by $W_{v}$, is achieved (here $\epsilon=10^{-10}$ ) or if the maximum number of iterations $N_{i t e r}^{\max }=350$ is reached. $M I B$ stands for the Minimum Impulse Bit (minimum shooting time that a thruster can execute), $\lambda=1.7$ allows to manage the convergence time and $M_{k}^{\ddagger}, k=1, \ldots, 16$ stands for the generalized inverse of $M_{k}, k=1, \ldots, 16$, optimal in the sense of the considered $p$-norm. The indices $k=1, \ldots, 16$ coincide with the indices of $\mathcal{S}_{k}$ given in (16) and (18).

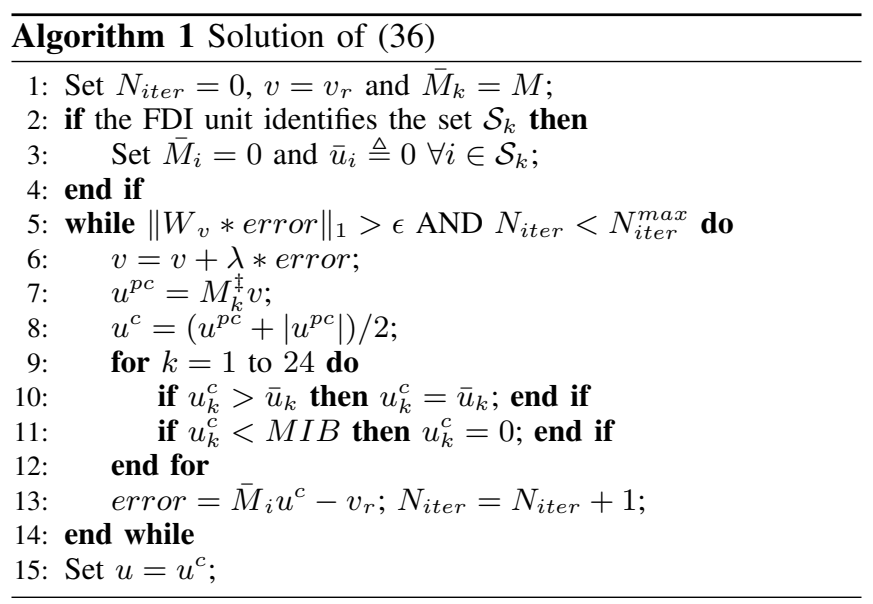

\section{SimUlation CAMPAIGN}

A simulation campaign is next conducted using the industrial high-fidelity simulator. Due to place limitation, only the synchronisation phase with faults occurring in thrusters $1,5,13,17$ are presented. A total of 450 runs have been done, 50 in fault free situations and 400 in faulty situations, the fault time occurrence being chosen randomly. As an illustration, Fig. 2 and 3 illustrate the behaviour of the attitude (in degrees) and relative position (in meters) for the case of stuck-open faults. The 50 fault free runs have been superposed (red plots).

Robustness and performance metrics are next established in terms of statistics, see Table II. The proposed metrics are $i$ ) 

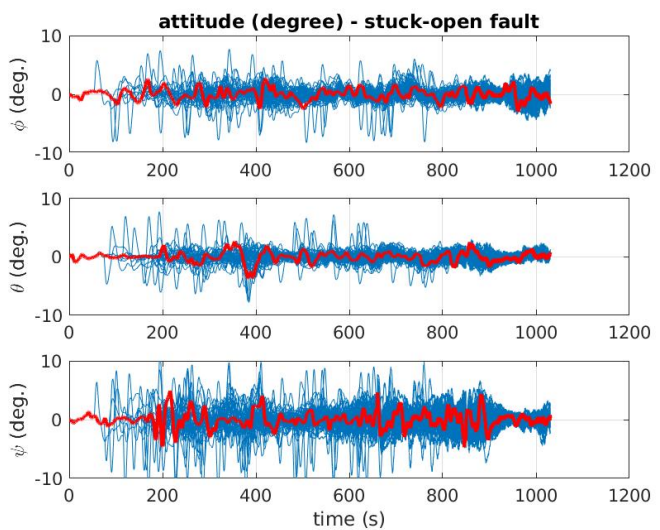

Fig. 2. Behaviour of the attitude (deg.) for 250 runs
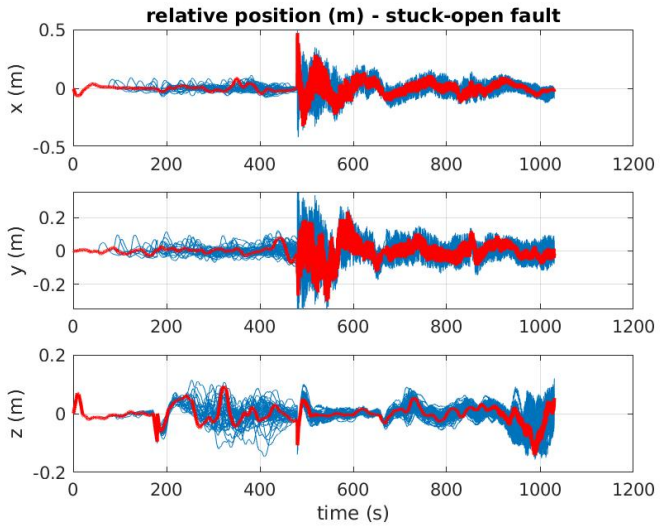

Fig. 3. Behaviour of the relative position (m) for 250 runs

the false alarm rate $\mathrm{FA} \%$, the true detection rate $\mathrm{TD} \%$ and the true recovery rate TRT\%; $i$ ) the diagnosis time performance (DTP) index which is used to quantify the diagnosis time performance; iii) $\Delta \Theta_{F D I}$ and $\Delta X_{F D I}$ which quantify, respectively, the maximum attitude error and the maximum relative position error, before it has been diagnosed by the FDI unit; iv) $\Delta \Theta_{F T C}$ and $\Delta X_{F T C}$ which quantify, respectively, the maximum attitude error and the maximum relative position error, before it has been accommodated by the FTC unit. These mission-oriented criteria enable to quantity the worst case of mission performance loss due to the faults. Note that these worst cases have been observed during transient behaviours. We argue that since the attitude, the angular velocity, the relative position and the relative velocity during the last $200 \mathrm{~s}$ are within their required value, the robotic arm can be deployed and the capture of ENVISAT can be done with success.

\section{CONCLUSION}

This paper discussed the design of a model-based FDIFTC unit for thruster faults that may occur in the propulsion unit of the chaser of the ESA's e.Deorbit mission, a mission whose aim is to remove the satellite ENVISAT from the Earth orbit. The proposed solution is based on a class of nonlinear unknown input observers and a modified version

\begin{tabular}{r|c|c|c} 
& no fault & stuck-open & stuck-closed \\
\hline FA\% & 0 & - & - \\
TD $\%$ & - & 100 & 100 \\
TRT\% & - & 100 & 100 \\
\hline \hline & & mean/std/max & mean/std/max \\
DTP(s) & - & $0.97 / 0.14 / 1.23$ & - \\
$\Delta \Theta_{F D I}(d e g)$ & - & $0.90 / 0.81 / 3.79$ & $1.47 / 0.95 / 4.45$ \\
$\Delta X_{F D I}(m)$ & - & $0.04 / 0.04 / 0.28$ & $0.07 / 0.09 / 0.35$ \\
$\Delta \Theta_{F T C}(d e g)$ & - & $8.19 / 1.89 / 9.97$ & $5.12 / 1.13 / 6.47$ \\
$\Delta X_{F T C}(m)$ & - & $0.27 / 0.1 / 0.5$ & $0.24 / 0.09 / 0.52$ \\
\hline
\end{tabular}

TABLE II

STATISTICS (WORST CASES)

of the nonlinear inverse pseudo control allocation technique. An intensive simulation campaign conducted within the industrial e.Deorbit simulator, demonstrates the efficiency of the approach.

\section{REFERENCES}

[1] H. Alwi, C. Edwards, and A. Marcos, "FDI for a mars orbiting satellite based on a sliding mode observer scheme," in Conference on Control and Fault-Tolerant Systems (SysTol). Nice, France: IEEE, 2010, pp. 125-130.

[2] EADS-Astrium, Deimos Space, and University of Leicester, Modern Control Techniques Applied to Satellite FDIR Final Presentation. Final presentation June 17th 2011, 2011.

[3] A. Falcoz, F. Boquet, and G. Flandin, "Robust $h_{\infty} / h_{-}$thruster failure detection and isolation with application to the lisa pathfinder spacecraft," in AIAA Guidance, Navigation, and Control Conference. Toronto, Ontario: AIAA, 2010.

[4] R. Fonod, D. Henry, C. Charbonnel, E. Bornschlegl, D. Losa, and S. Bennani, "Robust FDI for fault-tolerant thrust allocation with application to spacecraft rendezvous," Control Engineering Practice, vol. 42, pp. 12-27, 2015.

[5] P. Frank, E. Alcorta-Garcia, and B. Köppen-Seliger, "Modelling for fault detection and isolation versus modelling for control," Mathematical and Computer Modelling of Dynamical Systems, vol. 7, no. 1, pp. $1-46,2001$.

[6] D. Henry, "Fault diagnosis of the MICROSCOPE satellite actuators using $H_{\infty} / H_{-}$filters," AIAA Journal of Guidance, Control, and Dynamics, vol. 31, no. 3, pp. 699-711, 2008.

[7] D. Henry, J. Cieslak, A. Zolghadri, and D. Efimov, " $H_{\infty} / H_{-}$LPV solutions for fault detection of aircraft actuator faults: Bridging the gap between theory and practice," International Journal of Robust and Nonlinear Control, vol. 25, no. 5, p. start page 649, 2015.

[8] D. Henry, C. LePeuvédic, L. Strippoli, and F. Ankersen, "Robust model-based fault diagnosis of thruster faults in spacecraft," in SAFEPROCESS'2015. Paris, France: Ifac, 2015, pp. 1078-1083.

[9] H. Jin, P. Wiktor, and D. DeBra, "An optimal thruster configuration design and evaluation for quick step," Control Engineering Practice, vol. 3, no. 8, pp. 1113-1118, 1995.

[10] T. A. Johansen and T. I. Fossen, "Control allocationa survey," Automatica, vol. 49, pp. 1087-1103, 2013.

[11] M. Kerr, A. Marcos, L. Penin, and E. Bornschlegl, "Gain-scheduled FDI for a re-entry vehicle," in AIAA Guidance, Navigation and Control Conferences and Exhibit. Honoluku - Hawaii: AIAA, 2008, pp. AIAA-2008-7266.

[12] C. LePeuvédic, C. Charbonnel, D. Henry, L. Strippoli, and F. Ankersen, "Fault Tolerant Control Design for Terminal Rendezvous around Mars," in Proceedings of the 9th International ESA Conference on Guidance, Navigation \& Control Systems, Portugal, 2014, p. June.

[13] R. Patton, F. Uppal, S. Simani, and B. Polle, "Robust FDI applied to thruster faults of a satellite system," Control Engineering Practice, vol. 18, no. 9, pp. 1093-1109, 2010.

[14] C. Pittet, A. Falcoz, and D. Henry, "A model-based diagnosis method for transient and multiple faults of aocs thrusters," in 20th IFAC Symposium on Automatic Control in Aerospace ACA 2016, vol. 49. Sherbrooke, Quebec, Canada: IFAC, 2016, pp. 82-87.

[15] A. Posch, A. Schwientek, J. Sommer, and W. Fichter, "Modelbased on-board realtime thruster fault monitoring," in Proc. of IFAC Symposium on Automatic Control in Aerospace, Würzburg, Germany, 2013, pp. $553-558$ 